
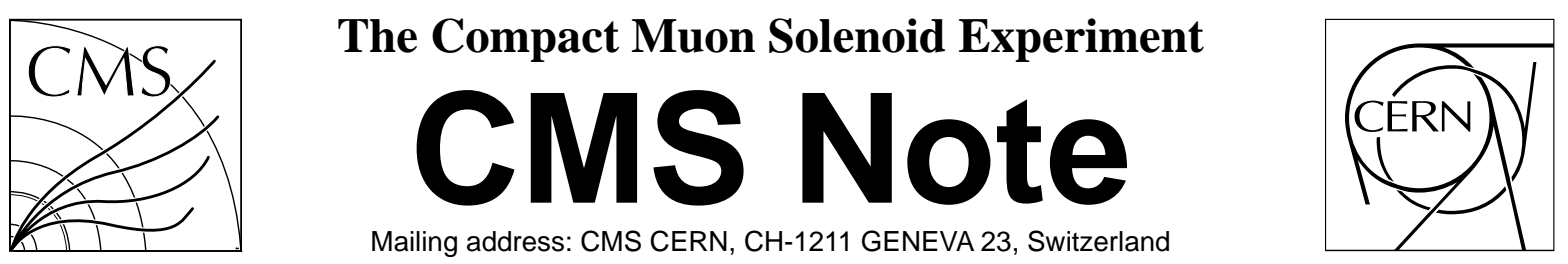

10 October 2000

\title{
Concept of the First Level Global Trigger for the CMS Experiment at LHC
}

\author{
Claudia-Elisabeth Wulz
}

Institute for High Energy Physics, Austrian Academy of Sciences, Nikolsdorfergasse 18, A-1050 Vienna, Austria

\begin{abstract}
The requirements, the principles and the design of the First Level Global Trigger Processor of the CMS experiment under construction at the CERN Large Hadron Collider are described. A trigger decision based on trigger objects delivered by the detector subsystems is provided for each beam crossing at a rate of $40 \mathrm{MHz}$. The Global Trigger comprises a novel concept where event selection is not only based on objects exceeding energy or momentum thresholds but also on complex event topology, which is made possible by the availability of space, charge and quality information in the algorithm calculations. The trigger logic is largely programmable in order to satisfy all possible physics requirements.
\end{abstract}




\section{Introduction}

CMS (Compact Muon Solenoid) [1] is a general-purpose experiment designed to study proton-proton and heavyion collisions at the Large Hadron Collider (LHC) of CERN. Its main feature is a strong solenoidal magnetic field ensuring high momentum resolution for charged particles. Apart from the superconducting coil [2] the apparatus consists of a silicon inner tracker with an embedded pixel detector [3, 4], a lead tungstate crystal electromagnetic calorimeter [5], a copper-scintillator sandwich hadron calorimeter [6] and a sophisticated four station muon system [7] featuring dedicated trigger chambers, the resistive plate chambers (RPC), and tracking chambers, which are also used in the trigger. The latter are made up of drift tubes with bunch crossing identification capability (DT) in the central region and cathode strip chambers (CSC) in the forward parts of the experiment. The RPC trigger chambers are mounted on the tracking chambers. To improve detector coverage a Forward Calorimeter consisting of a copper matrix with embedded quartz fibres is added in the forward regions [6]. A perspective view of the CMS experiment is shown in Fig. 1.

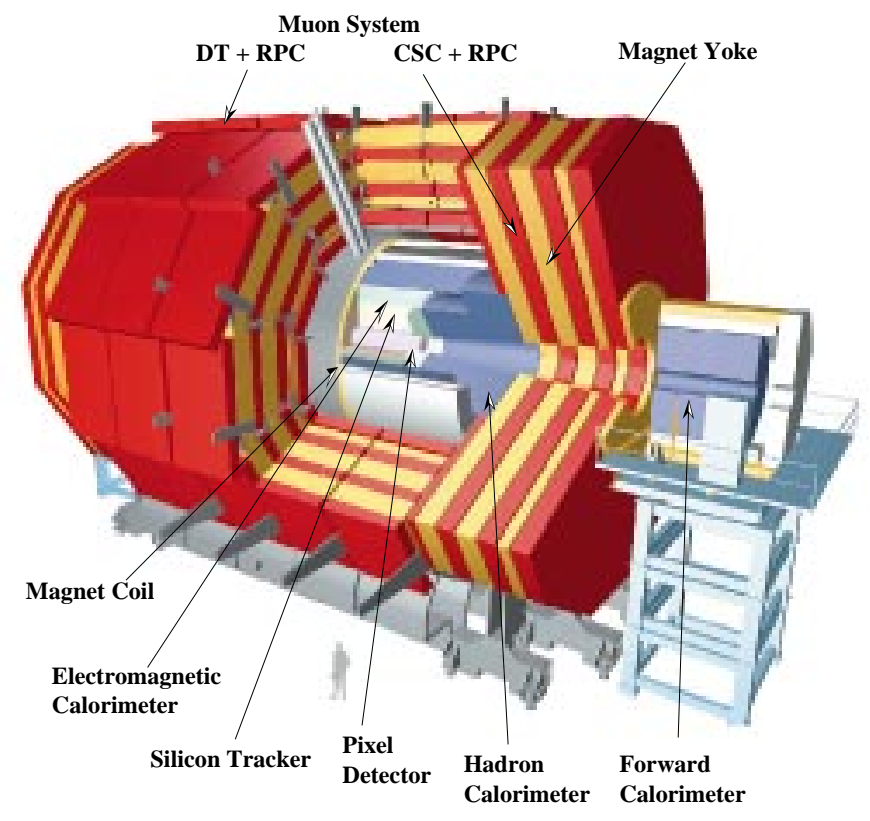

Figure 1: Perspective view of the CMS detector

The purpose of the trigger system of the experiment is the selection of all interesting events in the presence of an overwhelming background. Potentially interesting events at LHC are those including a Standard Model Higgs particle, supersymmetric or any other previously unknown particles as well as top events, $\mathrm{WW}, \mathrm{ZZ}, \mathrm{W} \gamma$ and many others. Background events originate from collisions that carry no new information for the study of matter and its origin. The trigger also has to select events used for calibration, synchronisation or testing purposes.

The trigger system of CMS is realized in several sequential stages. The First Level (L1) Trigger is a custombuilt electronics system since it has to take the decision to accept or reject an event in a short time, within a few microseconds after a collision. The Higher Level Trigger stages, including the Second Level Trigger, are implemented by software. They have much longer processing times and are based solely on commercial computer farms.

The Global Trigger, the subject of this publication, is the top level of the L1 Trigger System illustrated in Fig. 2. For physics data, decisions taken by the Global Trigger are based on the trigger objects delivered by the Global Calorimeter and Global Muon Triggers. Examples of trigger objects are candidate electrons, jets or muons. They are determined in three logical steps. The first one is the production of local trigger information, the so-called Trigger Primitives, by the calorimeters and the muon system. The following two steps are calculations of regional and global sub-detector specific quantities. For the RPC's it must be remarked that they do not have distinct local and regional trigger systems. They combine these two logical steps in a single one. The global quantities are forwarded to the Global Trigger. The trigger objects are based on coarsely segmented trigger data from the sub-detectors. The full-resolution data, which can be used by the Higher Level Triggers, are stored in pipeline 


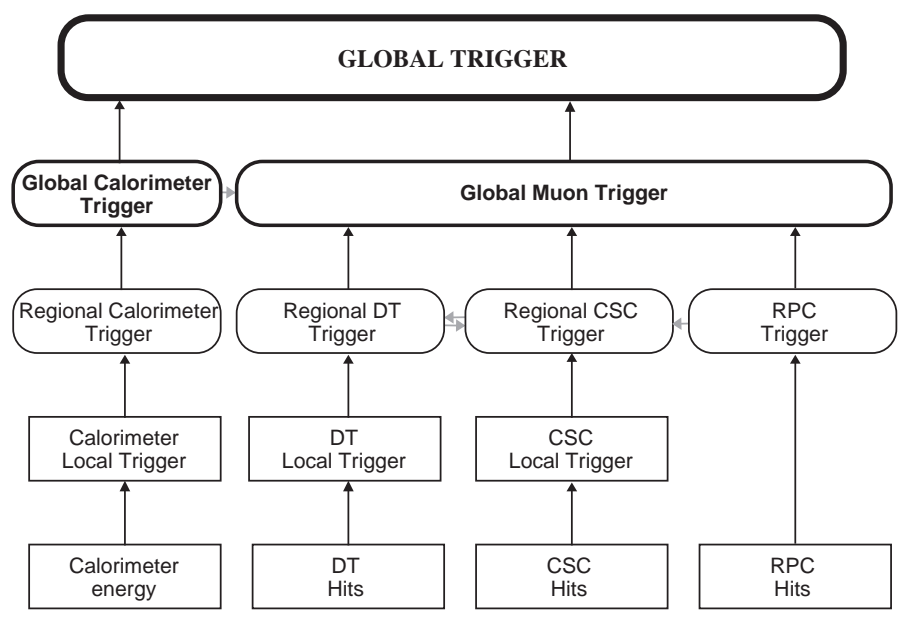

Figure 2: The CMS L1 Trigger System

memories in the front-end electronics. The Global Trigger can also use other signals for specific runs, such as calibration or test procedures.

The LHC's bunch crossing frequency for proton-proton interactions is $40 \mathrm{MHz}$ and the design luminosity is $10^{34} \mathrm{~cm}^{-2} \mathrm{~s}^{-1}$ [8]. At this luminosity approximately 25 inelastic collisions occur every $25 \mathrm{~ns}$ corresponding to an interaction rate of the order of $1 \mathrm{GHz}$. Interesting physics processes may, however, have small production rates. An example is the Standard Model Higgs particle which, for a mass of $\mathrm{m}_{H} \approx 120 \mathrm{GeV}$ and a decay mode into two photons, has a cross-section of only about $100 \mathrm{fb}$ giving rise to one Higgs event every thousand seconds at the design luminosity of the LHC. After the Higher Level Triggers the filtered event rate must not exceed $100 \mathrm{~Hz}$. This limit is driven by the capacity of the mass storage media and the on-line and off-line computing resources. The maximum output rate of the $\mathrm{L} 1$ trigger is $100 \mathrm{kHz}$, which is determined by the speed of the trigger electronics and the maximum rate that can be tolerated at the input of the Second Level Trigger. In practice, a maximum rate of $30 \mathrm{kHz}$ is foreseen in order to guarantee a large safety margin in case the projected event cross-sections for the proton-proton center of mass energy of $14 \mathrm{TeV}$ turn out to be underestimated. These two rates will be reduced to $75 \mathrm{kHz}$ and $25 \mathrm{kHz}$ respectively at the start-up of the LHC in 2005 or 2006 due to the deliberate incompleteness of the on-line processor farm which will be upgraded at a later stage in order to take advantage of technological progress. In addition, at the start-up of the accelerator the full design luminosity will not be available immediately.

The L1 Trigger electronics has to provide a decision to retain or to reject an event for each bunch crossing, every $25 \mathrm{~ns}$. It is pipelined and runs dead-time free by principle. However, subsystems may run into conditions that do not allow them to receive further L1 accept decisions during a certain time. The L1 Global Trigger is designed to take care of these situations as described in the next chapter. The trigger decision must occur at a fixed time after each interaction.

A basic principle of the CMS L1 Trigger is that, unlike most other collider experiments, it does not histogram objects exceeding energy or momentum thresholds. Trigger objects are sorted according to their rank determined from transverse energy or momentum and quality information. The highest rank objects are always sent to the Global Trigger, which then applies thresholds and other selection criteria according to physics or technical requirements. These criteria may be adapted as needed; there is no dependence on thresholds at local or regional levels. No cuts are applied at these levels except for inherent thresholds such as those necessary for the calculation of isolation information for electromagnetic clusters, the definition of a jet or the determination of jet multiplicities.

Another special and important feature of the CMS L1 Global Trigger is that it not only receives particle energies or momenta but also location information, notably pseudorapidity and azimuth. For muon candidates charge information is also delivered. Trigger conditions based on event topology can therefore be applied already at L1. Furthermore, the space coordinates can be used in the Higher Level Triggers to select regions of interest and therefore to save processing time. Spatial information is not only useful for physics triggers but also for detector calibration and trouble-shooting purposes. Comparable collider experiment L1 triggers [9-21] in general only apply conditions based on objects exceeding fixed energy or momentum thresholds and do not calculate spatial relations between them. At most trigger information arranged in an $\eta-\phi$ matrix structure is exploited using simple logical functions. 
Examples of basic physics trigger conditions using different combinations of electrons/photons, muons, jets, $\tau$ 's, total and missing transverse energies together with thresholds suitable for proton-proton luminosities up to $10^{34} \mathrm{~cm}^{-2} \mathrm{~s}^{-1}$ depending on the selected processes are shown in Table 1. Typical physics channels that can be explored with these trigger criteria are also shown. $\mathrm{H}_{S M}$ denotes the Standard Model Higgs, h, $\mathrm{H}, \mathrm{A}$ and $\mathrm{H}^{ \pm}$the Higgs particles of the Minimal Supersymmetric Standard Model, $t$ and $b$ stand for top and bottom quarks, lq for leptoquarks. The supersymmetric particles gluinos, squarks, sleptons, neutralinos and charginos are denoted as $\tilde{g}, \tilde{q}, \tilde{l}, \tilde{\chi}^{0}$ and $\tilde{\chi}^{ \pm}$respectively. W, W', Z and Z' are the Standard Model and heavy intermediate vector bosons. DY stands for Drell-Yan processes. $\Upsilon, \Upsilon$ ' and $\Upsilon^{\prime}$ " are the $b \bar{b}$ bound states whose suppression in the formation of a quark-gluon plasma is explorable with heavy-ion collisions. V's are heavy bosons required in some models of strongly interacting W's and Z's. $\rho_{T C}$ are similar objects appearing in technicolour models. B-physics triggers will normally only be used in the low luminosity regime up to $10^{33} \mathrm{~cm}^{-2} \mathrm{~s}^{-1}$. The Global Trigger thresholds need to be adjusted to deliver no more than the maximum Second Level input rate of $30 \mathrm{or} 25 \mathrm{kHz}$. In principle this rate should be subdivided equally between triggers involving the calorimeters and the muon system. However, since at a given output rate the efficiency to select real physics events from calorimeter and muon triggers will in general not be the same due to noise, pile-up or other factors, an adjustment under real running conditions will have to be made. Detailed rate calculations as a function of thresholds and luminosity may be found in [22]. Reasonable thresholds can be applied to keep the trigger rates within the defined limits.

Table 1: Typical trigger conditions and thresholds

\begin{tabular}{|c|c|l|}
\hline Trigger & Thresholds/GeV & Examples of explorable physics channels \\
\hline $1 \mu$ & 15 & $\mathrm{H}_{S M}, \mathrm{H}, \mathrm{A}, \mathrm{H}^{ \pm}, \mathrm{W}, \mathrm{W}, \mathrm{t}, \mathrm{B}-$ physics channels \\
$2 \mu$ & 5,5 & $\mathrm{H}_{S M}, \mathrm{~h}, \mathrm{H}, \mathrm{A}, \mathrm{Z}, \mathrm{Z}, \mathrm{DY}, \mathrm{V}, \rho_{T C}, \tilde{l}, \tilde{\chi}^{0}, \tilde{\chi}^{ \pm}, \mathrm{lq}, \psi, B_{s}^{0} \rightarrow 2 \mu, \Upsilon, \Upsilon$ \\
$\mu$ &,$\Upsilon^{\prime}$, \\
$\mu+e / \gamma$ & 5,15 & $\mathrm{H}_{S M}, \mathrm{H}, \mathrm{A}, \mathrm{t}, \mathrm{WW}, \mathrm{WZ}, \mathrm{W} \gamma, \tilde{l}, \tilde{\chi}^{0}, \tilde{\chi}^{ \pm}, \rho_{T C}, \mathrm{~V}$ \\
$\mu+$ jet(s) & 5,50 & $\mathrm{H}_{S M}, \mathrm{~h}, \mathrm{H}, \mathrm{A}, \tilde{g}, \tilde{q}, \mathrm{lq}, \mathrm{t}$ \\
$\mu+E_{T}^{\text {missing }}$ & 5,100 & $\mathrm{t}, \tilde{g}, \tilde{q}, \tilde{l}, \mathrm{lq}, \mathrm{WW}, \mathrm{WZ}, \mathrm{W} \gamma$ \\
$1 e / \gamma$ & 20 & $\mathrm{H}_{S M}, \mathrm{~h}, \mathrm{H}, \mathrm{A}, \mathrm{W}, \mathrm{W}, \mathrm{t}, \mathrm{B}-\mathrm{physics}$ channels \\
$2 e / \gamma$ & 15,15 & $\mathrm{H}_{S M}, \mathrm{~h}, \mathrm{H}, \mathrm{A}, \mathrm{Z}, \mathrm{Z}, \mathrm{DY}, \mathrm{WW}, \mathrm{WZ}, \mathrm{W} \gamma, \tilde{l}, \tilde{\chi}^{0}, \tilde{\chi}^{ \pm}, \mathrm{lq}$ \\
2 jets & 60,60 & $\mathrm{QCD}$ \\
$e / \gamma+$ jet(s) & 15,60 & $\mathrm{H}_{S M}, \mathrm{~h}, \mathrm{H}, \mathrm{A}, \tilde{g}, \tilde{q}, \mathrm{lq}, \mathrm{QCD}(\gamma+$ jets, $\mathrm{W}+$ jets $)$ \\
jets $+E_{T}^{\text {missing }}$ & 60,100 & $\tilde{g}, \tilde{q}, \mathrm{H}^{ \pm}$ \\
$\mu+\tau$ & 5,20 & $\mathrm{H}_{S M}, \mathrm{H}, \mathrm{A}, \tilde{g}, \tilde{q}$ \\
$e / \gamma+\tau$ & 15,20 & $\mathrm{H}_{S M}, \mathrm{H}, \mathrm{A}, \tilde{g}, \tilde{q}$ \\
$\tau+$ jets & 15,60 & $\mathrm{H}^{ \pm}$ \\
\hline
\end{tabular}

The ultimate goal of the L1 Global Trigger System is the generation of the L1 Accept/Reject (L1A) signal and its transfer to the Timing, Trigger and Control (TTC) System [23], which in turn broadcasts it to the different subdetector front-end controllers using high power laser sources that fan out the signals via an optoelectronic multiplexed distribution network. The Global Trigger must work dead time free by itself and provide a trigger decision every $25 \mathrm{~ns}$. Due to speed and dataflow requirements it has to run in pipelined mode, synchronously with the LHC clock. However, due to readout limitations of some subsystems, trigger rules permitting no more than a certain number of triggers within a given number of bunch crossings have to be established. These rules have to be set such that the overall dead time stays well below $1 \%$.

The trigger decision has to be taken after a fixed latency of $3.2 \mu$ s corresponding to a delay of 128 bunch crossing intervals. This is determined by the present technical limitations in the depth of the analog pipeline memories used by certain subsystems. Safe operation in the harsh LHC radiation environment is a key requirement, therefore the location of the Global Trigger electronics is on average $90 \mathrm{~m}$ away from the detector, in the counting room adjacent to the experimental hall in the underground cavern. Signal propagation and distribution times consequently represent a significant fraction of the overall latency. Drift times in the muon chambers also account for up to 16 bunch crossing intervals. Not much more than one microsecond is available for the actual processing of the full L1 Trigger. This includes the generation of trigger primitives such as local energy depositions in the calorimeters or track stubs in the muon system and the calculation time for the subsequent algorithms. The Global Trigger, being the last stage of the L1 trigger chain, has to wait until all subdetectors have delivered their trigger information and therefore has to perform its calculations in a much shorter time, within a quarter of a microsecond.

A block diagram of the L1 Global Trigger environment containing the directions of information exchange is shown in Fig. 3. For normal physics running the Global Trigger Processor uses information from the calorimeters and the 
muon system only. Should input from the inner tracking system become feasible in the future, the Global Trigger may eventually include it in its operation. For example, it could use data from the pixel detector surrounding the beam pipe opening up the possibility to recognize multiple vertices at the lowest level of the trigger chain. For non-physics running the Global Trigger is also able to use special signals directly fed into its electronics for setup, calibration, synchronisation and testing purposes. These triggers are labeled as technical triggers.

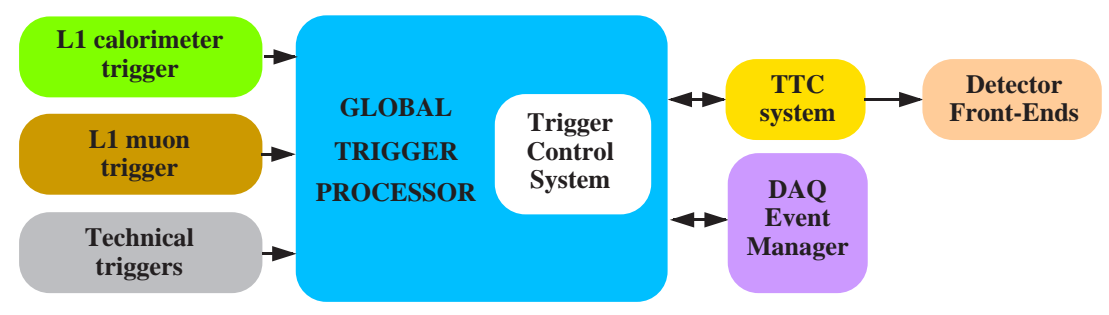

Figure 3: CMS L1 Global Trigger environment

The heart of the Global Trigger Processor is the logic performing the trigger algorithm calculations. An algorithm is a combination of trigger objects satisfying defined threshold, space and quality requirements. For physics runs up to 128 different algorithms are possible. The basic principle is that all of them are processed in parallel, and the results are delivered as the trigger output word - a string of 128 bits each representing one algorithm. The L1 Accept/Reject decision is then taken according to the run-specific trigger setup word predefined by physicists and transmitted by the run control part of the Data Acquisition System. The input word is also a 128-bit string, which in general has fewer bits set than the trigger output word since the algorithm calculations are performed regardless of the trigger setup. The 128 trigger algorithm conditions are largely programmable but are expected to be stable after the running-in phase of the experiment. It will, however, always be possible to adapt algorithm conditions if interesting unforeseen experimental signatures appear. Each algorithm has a separate rate counter and can be prescaled. This is essential for recording high rate data samples and for checking trigger efficiencies. Vetoing certain triggers is also possible. The TTC System, apart from distributing the L1 Accept/Reject decision, transmits control signals such as the $40 \mathrm{MHz}$ LHC machine clock, the Bunch Crossing Zero (BC0) or the Bunch Counter Reset (BCR) signals. The LHC orbit structure is depicted in Fig. 4. The total length of an orbit is 3564 bunch crossing intervals corresponding to a time of approximately $89 \mu \mathrm{s}$. At the end of the cycle there is a period of 119 missing bunch crossing intervals corresponding to $3 \mu$ s without proton collisions, the abort gap, which may be used for purposes such as calibration, resynchronisation, resetting or testing. There are also shorter gaps interleaved in the cycle, which could be used as well.

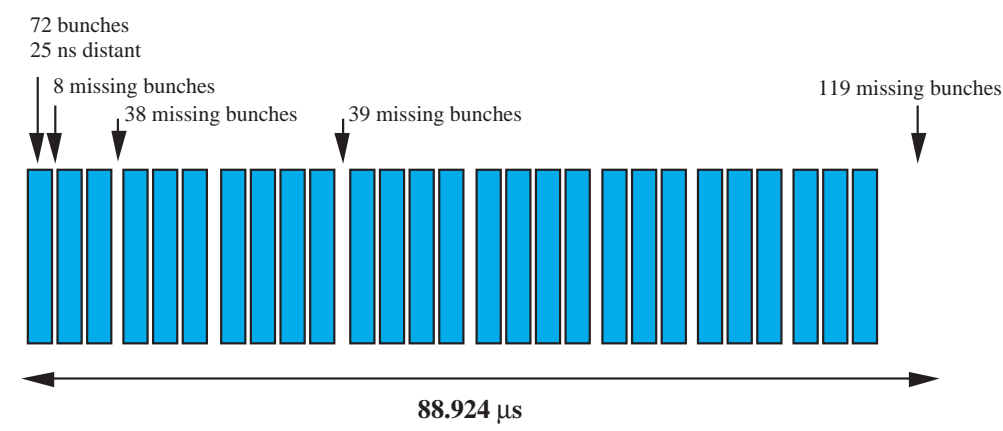

Figure 4: LHC orbit structure

The main task of the Trigger Control System (TCS) is to ensure that the subsystems are ready to receive the L1A coming from the Global Trigger. It therefore controls the delivery of L1A signals according to the status of the readout electronics or the data acquisition which is determined by state machines emulating the occupancy of the subdetector front-end pipeline buffers or by direct warning or even error signals through a fast monitoring network. In parallel it guarantees that the sequence of L1 Accepts adheres to the trigger rules described above. The part of the electronics taking care of rate control is called the Trigger Throttle System (TTS).

The TCS is furthermore responsible to generate the $\mathrm{BC} 0$ and reset commands. It is logically located between the 
Global Trigger and the Readout and Data Acquisition (DAQ) Systems. Physically it is incorporated partly in the Global Trigger crate, partly in the TTC electronics.

The TCS distributes its information directly to the DAQ Event Manager and via the TTC System to the detector front-end electronics. The Event Manager controls the flow of events. It receives the front-end event identifiers from the TCS and sends status information back to it via the fast monitoring network. The L1 trigger system consists of custom-built electronics housed in a single $9 \mathrm{U}$ high VME crate. The electronic modules of the Global Trigger Processor are built in FPGA technology. ASIC's have not been chosen for several reasons: The most important aspects determining the choice were the required flexibility and adaptability of the algorithm logic calculations in order to be ready to fully exploit new physics. The speed requirements can already be met by today's gate arrays and the number of identical chips is not large. Independence from ASIC manufacturing technologies was also a criterion for the decision. The backplane is fully custom built.

\section{Conceptual Design}

In the following the input to the Global Trigger, the algorithm and decision logic and the communication with the TTC and DAQ systems are described. The part of the Global Trigger belonging to the TCS is described elsewhere [24].

\subsection{Input}

The algorithm calculations and the delivery of the L1A signal are not the only tasks of the CMS Global Trigger. An important first step is the time-alignment of the trigger data arriving from the subsystems since they arrive with different offsets at the input of the Global Trigger. Whilst the information from the Global Calorimeter Trigger arrives after 85 bunch crossing intervals, the data from the Global Muon Trigger, which are sent via the Global Trigger backplane, arrive only after 91 bunch crossings. The muon data are already synchronised to the LHC orbit. The calorimeter data still have to be synchronised and delayed in order to arrive at the same time as the muons at the Global Trigger logic. Since the latency is fixed for each subsystem it can be guaranteed that only input data originating from the same collision arrive at the Global Trigger.

The input modules are called the Pipelined Synchronising Buffer (PSB) cards. They receive the trigger objects from the subsystems by Channel Links ${ }^{\circledR}$. There are also dedicated PSB modules receiving fast control information such as ready, busy, warning or error signals. Input for technical triggers also goes to these boards, which logically belong to the Trigger Control System. Twelve input channels are available on each card. Synchronisation is performed with a synchronisation pipeline.

In the following the input for physics triggers, specifically the information coming from the calorimeters and the muon system, is described (see also Fig. 2). For the calorimeters local trigger information refers to energy depositions in the trigger towers of the electromagnetic and hadron calorimeters. The Regional Calorimeter Trigger $[25,26]$ then uses these Trigger Primitives to find candidate electrons or photons, jets and isolated hadrons from the decay of $\tau$ 's and to calculate transverse energy sums in different detector regions. The jet trigger uses the transverse energy sums of $12 \times 12$ electromagnetic and hadronic trigger towers covering a region of $\Delta \eta \times \Delta \phi=1.04 \times 1.04$. $\tau$-jets are distinguished from normal jets by requiring that none of the nine $4 \times 4$ sub-regions have a $\tau$-veto bit set. A $\tau$-veto bit is set on if there are more than two active towers in the $4 \times 4$ sub-region. All calorimeter trigger objects described so far are forwarded to the Global Calorimeter Trigger [27] which sorts the electrons or photons, $\tau$ 's and jets according to energy and quality and sends the four objects with the highest rank in each category to the Global Trigger. Furthermore, it determines the total and missing transverse energies as well as eight numbers representing jet multiplicities for eight different transverse energy thresholds, two of which are reserved for the forward region, and sends them to the Global Trigger. The crates of the Global Calorimeter Trigger are located next to the Global Trigger Crate to minimize transfer paths.

Concerning muons the situation is more complicated as CMS has an elaborate muon system. As already mentioned in the Introduction, there are special dedicated trigger chambers in addition to the tracking chambers, which also take part in the trigger. The resistive plate trigger chambers produce a pattern of hits, the barrel drift tube and forward cathode strip chambers (DT/CSC) deliver track vectors at each station. The RPC's cover both the barrel and the forward regions up to a pseudorapidity of $|\eta|=2.4$ (2.1 at the start-up of LHC). The Pattern Comparator Trigger (PACT) $[7,28]$ combines their hits to tracks.

The barrel drift tube chambers consist of three superlayers, each of which is composed of four staggered layers of drift cells. The wires of the inner and outer superlayers are parallel to the beam direction and measure the 
azimuthal coordinate $\phi$ in the magnetic bending plane. The central superlayer has wires orthogonal to the beam line and measures pseudorapidity $\eta$, i.e. the track position along the beam. The Bunch and Track Identifier (BTI) logic of a barrel drift tube chamber [29] finds track segments from coincidences of aligned hits in the four layers of one drift tube superlayer. For each chamber a Track Correlator [30] combines the track segments in the inner and outer superlayers and forwards them together with segments found in the central superlayer to the Trigger Server [31], which then sends the best two track segments in the $(r, \phi)$-plane and a bit pattern representing the segments in $\eta$ to the DT Regional Muon Trigger [32-36].

For the CSC's the Trigger Primitives are local charged tracks (LCT's) measured with cathode strips combined with anode wire information for bunch crossing identification [7, 37, 38]. The best three LCT's per chamber are transmitted to the CSC Regional Muon Trigger [39].

The Regional Muon Trigger is also called the Track Finder. It is made up of two separate parts running in parallel, one for the DT's and one for the CSC's. There is some information exchange between them in the overlap region joining the barrel and the endcaps. The Track Finder reconstructs muons out of the track segments found in the four muon stations. They are then sorted according to criteria that take into account transverse momentum and quality. The best four DT and the best four CSC muons are sent to the Global Muon Trigger (GMT) [7, 40], which combines them with the top eight muons found by the PACT of the RPC's and performs a DT/CSC-RPC matching based on transverse momentum, distance in $(\eta, \phi)$-space and quality. It then determines the best four muon candidates in the entire CMS detector, which are finally sent to the Global Trigger. The GMT greatly improves the overall muon trigger efficiency and rate capability compared to the individual muon subdetectors. For each muon, the sign of the electric charge, isolation information and an indication whether there is compatibility with an energy deposition delivered by a minimum-ionizing particle (MIP) are available at the level of the GMT. The latter two quantities are transmitted from the Regional Calorimeter Trigger through the Global Calorimeter Trigger via Channel Links ${ }^{\circledR}$ to the PSB cards belonging to the Global Muon Trigger. The connection between these and the GMT logic boards, which perform the correlation of the calorimeter MIP and isolation information with the muon candidates and add the relevant bits, is made with point-to-point links on the backplane. In total there are $252 \mathrm{MIP}$ and 252 isolation bits. The Regional Muon Trigger sends its muon candidates directly to the GMT boards, not via the PSB cards.

The details of the bit assignments for the Global Calorimeter and Global Muon Trigger links to the Global Trigger are listed in Table 2. The calorimeters send the four highest rank non-isolated and isolated electrons/photons,

Table 2: Bit Assignments for GCT and GMT Links to Global Trigger

\begin{tabular}{|c|c|}
\hline Data & Bit assignment \\
\hline Non-isolated $e / \gamma(1$ to 4$)$ & $\begin{array}{l}\mathrm{E}_{T}(0: 5), \eta(6: 9), \phi(10: 14) \\
\mathrm{P} 0(16), \mathrm{P} 1(17), \mathrm{SYN}(18), \mathrm{B} 0(19), \mathrm{B} 1(20)\end{array}$ \\
\hline Isolated $e / \gamma(1$ to 4$)$ & $\begin{array}{l}\mathrm{E}_{T}(0: 5), \eta(6: 9), \phi(10: 14) \\
\mathrm{P} 0(16), \mathrm{P} 1(17), \mathrm{SYN}(18), \mathrm{B} 0(19), \mathrm{B} 1(20)\end{array}$ \\
\hline Central jets (1 to 4$)$ & $\begin{array}{l}\mathrm{E}_{T}(0: 5), \eta(6: 9), \phi(10: 14) \\
\mathrm{P} 0(16), \mathrm{P} 1(17), \mathrm{SYN}(18), \mathrm{B} 0(19), \mathrm{B} 1(20)\end{array}$ \\
\hline Forward jets ( 1 to 4$)$ & $\begin{array}{l}\mathrm{E}_{T}(0: 5), \eta(6: 9), \phi(10: 14) \\
\mathrm{P} 0(16), \mathrm{P} 1(17), \mathrm{SYN}(18), \mathrm{B} 0(19), \mathrm{B} 1(20)\end{array}$ \\
\hline$\tau$-jets ( 1 to 4$)$ & $\begin{array}{l}\mathrm{E}_{T}(0: 5), \eta(6: 9), \phi(10: 14) \\
\mathrm{P} 0(16), \mathrm{P} 1(17), \mathrm{SYN}(18), \mathrm{B} 0(19), \mathrm{B} 1(20)\end{array}$ \\
\hline Total $\mathrm{E}_{T}$, bits $(3: 5)$ of $\phi$ - $\mathrm{E}_{T}^{\text {missing }}$ & $\begin{array}{l}\Sigma \mathrm{E}_{T}(0: 11), \mathrm{OV}(12), \phi-\mathrm{E}_{T}^{\text {missing }}(13: 15) \\
\mathrm{P} 0(16), \mathrm{P} 1(17), \mathrm{SYN}(18), \mathrm{B} 0(19), \mathrm{B} 1(20)\end{array}$ \\
\hline Missing $\mathrm{E}_{T}$, bits $(0: 2)$ of $\phi-\mathrm{E}_{T}^{\text {missing }}$ & $\begin{array}{l}\mathrm{E}_{T}^{\text {missing }}(0: 11), \mathrm{OV}(12), \phi-\mathrm{E}_{T}^{\text {missing }}(13: 15), \\
\mathrm{P} 0(16), \mathrm{P} 1(17), \mathrm{SYN}(18), \mathrm{B} 0(19), \mathrm{B} 1(20)\end{array}$ \\
\hline Jet counts ( 1 to 4$)$ & Count $1(0: 3)$, Count $2(4: 7)$, Count $3(8: 11)$, Count $4(12: 15)$ \\
\hline Jet counts $(5$ to 8$)$ & Count $5(0: 3)$, Count $6(4: 7)$, Count 7 (8:11), Count 8 (12:15) \\
\hline Muons ( 1 to 4$)$ & $\begin{array}{l}\phi(0: 7), \mathrm{p}_{T}(8: 12), \text { quality }(13: 15), \eta(16: 21) \\
\operatorname{sign}(22), \operatorname{MIP}(23), \operatorname{ISO}(24), \operatorname{SYN}(25)\end{array}$ \\
\hline
\end{tabular}

four central and four forward jets, four $\tau$-candidates, the total transverse energy and the azimuthal direction and magnitude of the missing transverse energy as well as the jet counts for eight different transverse energy thresholds. $\mathrm{E}_{T}$ and $\mathrm{p}_{T}$ denote transverse energy and momentum. The numbers in brackets are the bit numbers. P0, P1 are parity bits, SYN is a bit for synchronisation checks, and B0, B1 are bunch counter bits sent by the calorimeter 
trigger for phase checking purposes. OV represents an overflow bit for the total and missing transverse energies. Bit 15 is always set to zero for electrons, jets and $\tau$-jets. From the table one can see that the Global Trigger has to handle close to 600 bits, not counting the approximately 500 MIP and isolation bits for the Global Muon Trigger.

\subsection{Algorithm Logic}

The input for the physics trigger algorithm calculations are the trigger objects ordered by rank described in the previous chapter. In total there are 28 input channels consisting of groups of four muons, four isolated and four non-isolated electrons or photons, four central and four forward jets, four $\tau$-jets and four inputs with the transverse energy information and the jet counts as detailed in Table 2. Another quadruplet of channels could be accommodated in the future if needed.

An algorithm is defined as a logic combination of the trigger objects together with a set of energy or momentum thresholds, windows in $\eta$ and/or $\phi$ and topological conditions. All threshold and space parameters, except for some exceptions described above, are only applied at the Global Trigger stage. The transverse momentum and energy thresholds can be changed easily by VME instructions.

The first step in the algorithm logic consists of applying conditions to each group of objects. Energy or momentum thresholds and windows for the space coordinates are applied. Windows may of course be set to trivial values if they are not required. This will normally be the case for the azimuthal angle. $\phi$-Windows could, however, be useful for trouble-shooting purposes in case of localized detector problems. The pseudorapidity windows give the possibility to apply different thresholds in the central and forward regions. This is useful for instance in physics processes where forward tagging jets are required in the event selection such as in the case of Higgs particle production by vector boson fusion or in the investigation of a possible strong interaction regime in $\mathrm{WW}$ and $\mathrm{ZZ}$ scattering. For muons the isolation bit and the quality bits are also checked. For the quadruplet containing the transverse energies and jet counts thresholds are applied for each object. For the missing transverse energy vector $\phi$-windows can be applied. Distances in $\eta$ and $\phi$ between the objects are also calculated. For chip size reasons only "close" and "opposite" conditions are considered. If more precise spatial correlations are required, they can easily be taken care of by the Higher Level Triggers. For muons sign and MIP bit templates are also applied in this step. Different conditions may freely be selected for each of the trigger objects. The conditions concerning only objects of the same group are denoted as Particle Conditions. The conditions relating different types of objects, for example a muon and the missing transverse energy vector, are called Delta Conditions.

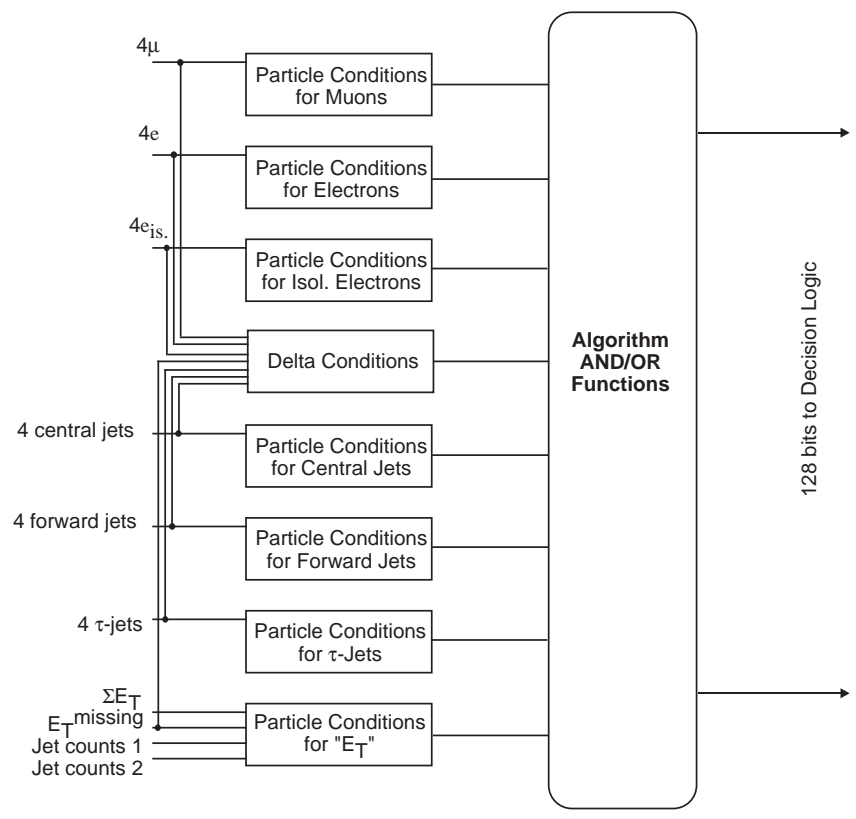

Figure 5: Algorithm logic

The second logical step is the actual algorithm calculation. A trigger algorithm is an AND-OR function using the Particle- and Delta Conditions as input. Any Condition can be used either as a trigger or a reject requirement. 128 
algorithms are available corresponding to 128 trigger or algorithm bits. Most of them will be used for physics running, but some are reserved for special algorithms such as a random trigger or special synchronisation triggers. All 128 algorithm calculations run in parallel. They are predefined and are always performed regardless of the trigger setup.

Fig. 5 shows a functional diagram of the algorithm logic, which resides on the Global Trigger Logic (GTL) boards. In the hardware design [41] the Delta and Particle Conditions (Muon-, Electron-, Isolated Electron-, Central Jet-, Forward Jet-, $\tau$ - and " $\mathrm{E}_{T}$ "-Conditions, which include also the treatment of the jet counts) as well as the algorithm calculations are actually not performed in distinct chips, but combined into a few making optimal use of the FPGA capacity. For conncectivity reasons not all theoretically possible Delta Conditions are calculated, which represents no restriction in practice.

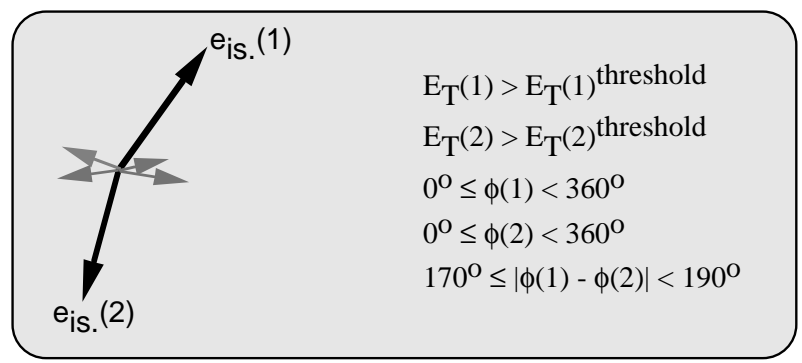

Figure 6: Particle Condition for back-to-back isolated electrons

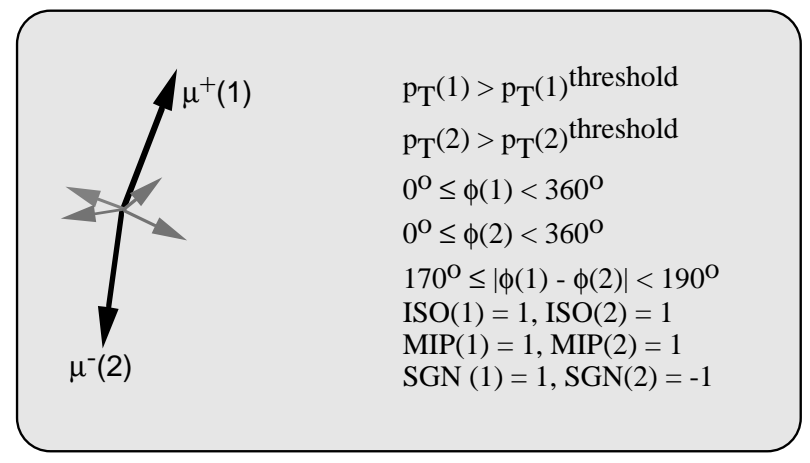

Figure 7: Particle Condition for back-to-back opposite sign isolated muons

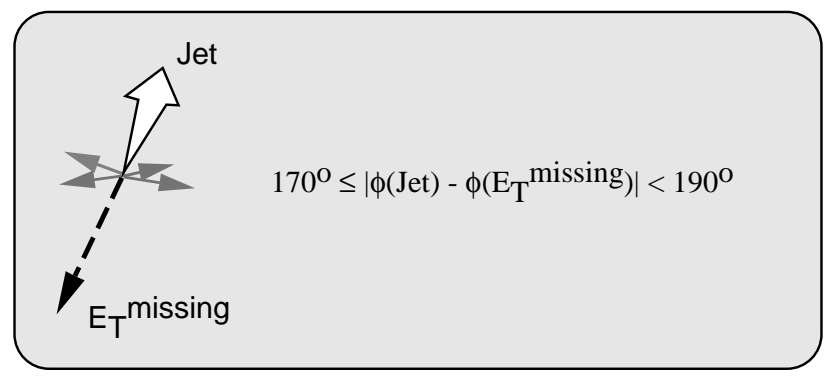

Figure 8: Delta condition for a jet opposite to the missing transverse energy vector

To illustrate the trigger logic further, examples of the steps necessary for the calculation of an algorithm are given. The first example is the use of a Particle Condition with a space correlation necessary to trigger on two isolated electrons back-to-back in azimuth, a signal which might come from the decay of a heavy vector boson (Z'). Fig. 6 shows a pictorial representation and the used mathematical relations. Physically reasonable values for the thresholds and other conditions are indicated, not those actually used in the hardware. Fig. 7 shows the case of two isolated muons of opposite charge back-to-back in azimuth, which could also come from a Z'. Here MIP and sign 
templates are applied in addition to the threshold and spatial requirements as for the electron case. An example for a Delta Condition is shown in Fig. 8, which depicts the case of a monojet event. A single jet is required to be opposite in $\phi$ to the missing transverse energy vector. Algorithm calculations are illustrated in Figures 9 and 10. In the first figure an isolated electron is required to appear in combination with missing transverse energy. Both objects must pass the requirements set in the respective Particle Conditions. In Fig. 10 an algorithm requiring two muons or two electrons exceeding certain transverse momentum or energy thresholds in conjunction with missing transverse energy, as could be the case for a SUSY slepton, is depicted.

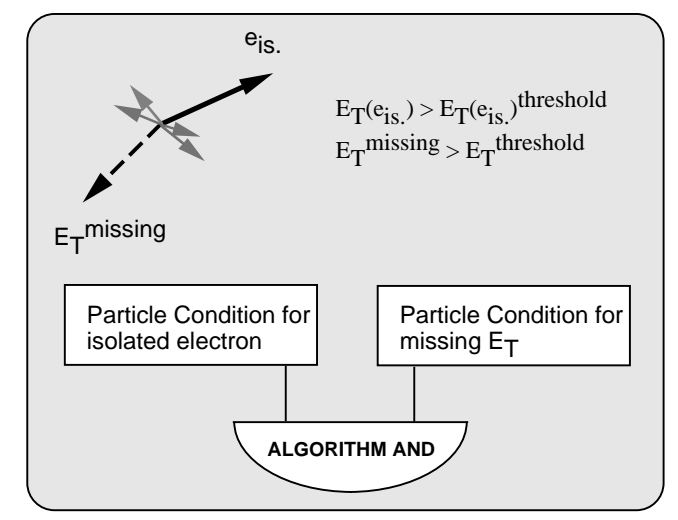

Figure 9: Algorithm for an isolated electron and missing transverse energy

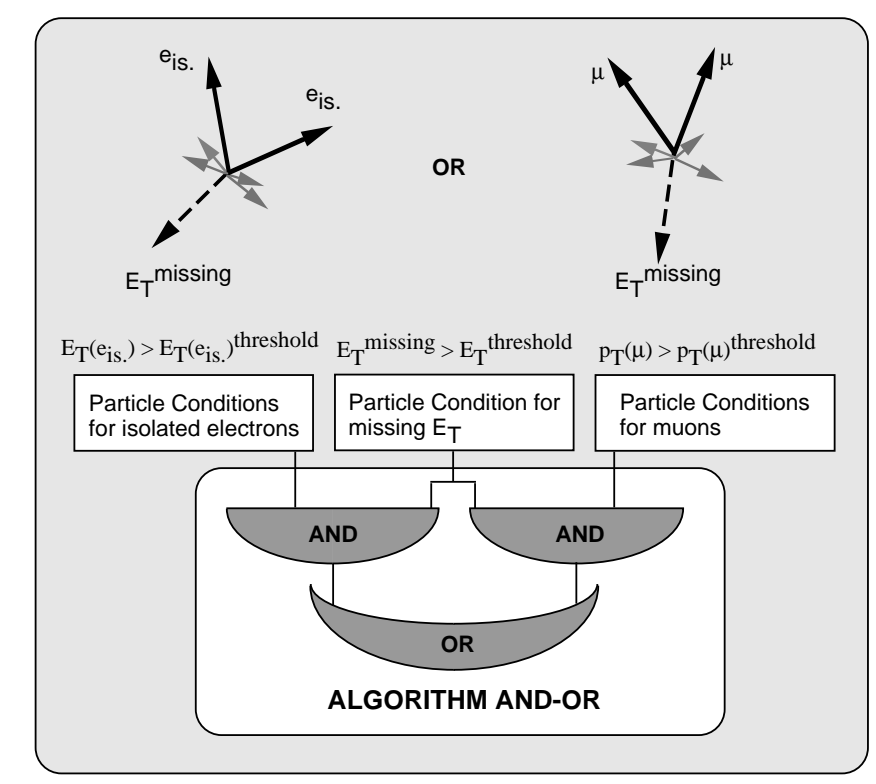

Figure 10: Algorithm for two muons or two electrons with missing transverse energy

\subsection{Decision Logic}

The L1A decision is taken by applying a final OR to the 128 previously calculated algorithm bits. The trigger setup is defined by run control. The decision logic is built into the Final Decision Logic (FDL) board, which also contains the programmable prescaling factors and the rate counters for each of the algorithms. Normally a single final OR is sufficient. For commissioning, calibration and other special procedures, however, it is desirable that subsets of the CMS detector can run independently. Therefore the different detector parts are arranged in "trigger partitions". Examples of partitions are the barrel pixel detector or one of the endcap CSC's. The DAQ Event Manager is also a distinct partition. There is a maximum of 32 partitions, which can either run in groups or separately. Within a partition group the event numbers are identical. A partition not taking part in the run sends an empty event record in order to keep synchronisation. For routine physics data taking there is naturally 
only one single partition group consisting of all the CMS subsystems. Up to eight final OR's are available for physics running to avoid latency problems. This is not considered to be a limitation, since it will be rare that eight partitions need to take different physics data concurrently. An example of two final OR's based on real physics events is the case where all detectors take normal physics data, but the electromagnetic calorimeter wishes also to accept W-events for calibration purposes. For each partition a 4-bit trigger type indicating whether the event was a physics, calibration or other kind of event is sent to the Event Manager. This facilitates the task for the Higher Level Triggers and the data acquisition since different processing or recording streams can be selected. The possibility to send an 8-bit L1 command word along with the L1A, which the subsystems can use to start standard or special procedures according to their needs, is presently under discussion.

\subsection{Communication with the TTC System and the Data Acquisition}

The communication with the TTC system is done with the Timing Module (TIM) which contains the timing receiver chip (TTCrx). After an L1A request it broadcasts a readout request to all modules in the Global Trigger crate. On each module there is a Readout Processor (ROP) collecting the trigger data, which are then formatted and transferred to the Global Trigger Front-End (GTFE) module. On this board the data from the different modules are merged and checked. A formatted Global Trigger event record is built and sent to the DAQ Event Manager using a standard CMS readout interface. The Global Trigger event record contains at least the event number, all input bits, the trigger output word with the 128 algorithm bits and the L1A decision. More information for monitoring and debugging purposes can be added if desired. The option to record events before and after the triggered event is also foreseen in order to keep trace of the trigger history. This is especially useful to control pile-up effects or noisy channels.

\section{Summary and Outlook}

The conceptual design of the CMS L1 Global Trigger has been presented. Its basic tasks are:

- Alignment of the arrival times of the subsystem trigger data,

- Algorithm calculations,

- L1 Accept decision,

- Communication with the Timing, Trigger and Control System,

- Communication with the Data Acquisition System.

According to these tasks the Global Trigger is composed of five functional units:

- Pipelined Synchronising Buffer (PSB) cards,

- Global Trigger Logic (GTL) cards,

- Final Decision Logic (FDL) card,

- Timing (TIM) card,

- Global Trigger Front-End (GTFE) card.

Fig. 11 shows the functional blocks of the Global Trigger processor and its interconnections. The Global Trigger

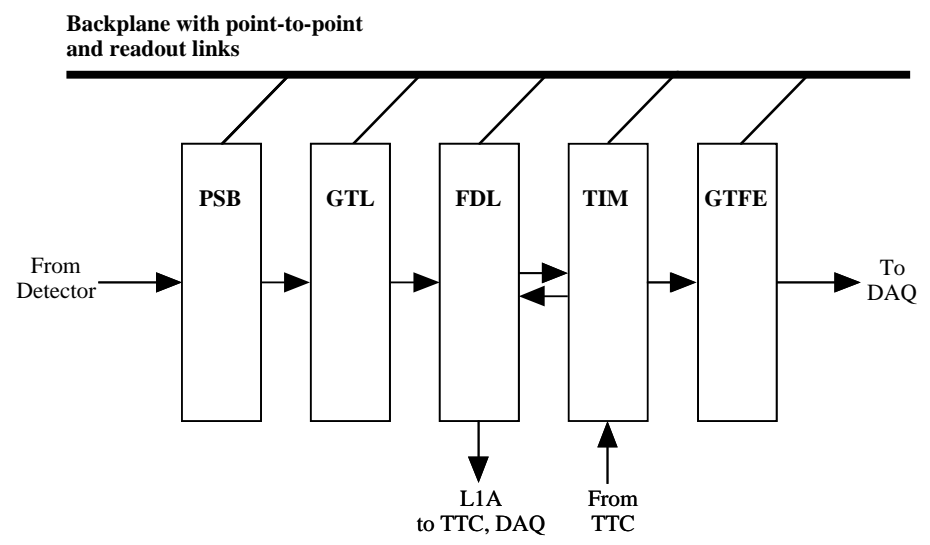

Figure 11: Functional blocks of the CMS Global Trigger Processor 
electronics built in FPGA technology fits into a single $9 \mathrm{U} \times 400 \mathrm{~mm}$ VME crate. Working prototypes for the crucial components, the PSB, the GTL boards and the custom backplane are available. The description of the hardware implementation of the Global Trigger and the prototypes may be found in [41]. The complete CMS L1

Global Trigger Processor is on schedule to be ready well before the start-up of the LHC.

\section{Acknowledgements}

The author gratefully acknowledges support by her colleagues, especially by A. Taurok, who is in charge of building the Global Trigger electronics, W.H. Smith, D. Denegri and J. Varela. Editorial assistance by her coworkers M. Brugger, M. Fierro and H. Rohringer of the Austrian CMS group and by F. Szoncsó was also greatly appreciated.

\section{References}

[1] CMS Technical Proposal, CERN/LHCC 94-38 (1994).

[2] The CMS Magnet Project - Technical Design Report, CERN/LHCC 97-10 (1997).

[3] The CMS Tracker Project - Technical Design Report, CERN/LHCC 98-6 (1998).

[4] Addendum to the CMS Tracker Technical Design Report, CERN/LHCC 2000-016 (2000).

[5] The CMS Electromagnetic Calorimeter Project - Technical Design Report, CERN/LHCC 97-33 (1997).

[6] The CMS Hadron Calorimeter Project - Technical Design Report, CERN/LHCC 97-31 (1997).

[7] The CMS Muon Project - Technical Design Report, CERN/LHCC 97-32 (1997).

[8] The Large Hadron Collider - Conceptual Design, CERN/AC/95-05 (1995).

[9] M. Campbell et al., "The CDF Trigger", Nucl. Instr. Meth. A265 (1988) 326.

[10] M. Abolins et al., "The Fast Trigger for the D0 Experiment", Nucl. Instr. Meth. A289 (1990) 543.

[11] D. Buskulic et al., "Performance of the ALEPH Detector at LEP", Nucl. Instr. Meth. A360 (1995) 481.

[12] V. Bocci et al., "Architecture and Performance of the DELPHI Trigger System", Nucl. Instr. Meth. A362 (1995) 361.

[13] V. Bocci et al., "Basic Concepts and Architectural Details of the DELPHI Trigger System", IEEE Trans. Nucl. Sci. 42 (1995) 837.

[14] R. Bizzarri et al., "The first level energy trigger of the L3 experiment", Nucl. Instr. Meth. A317 (1992) 463.

[15] P. Béné et al., "First-level charged particle trigger for the L3 detector", Nucl. Instr. Meth. A306 (1991) 150.

[16] M. Arignon et al., "The Trigger System of the OPAL Experiment at LEP", Nucl. Instr. Meth. A313 (1992) 103.

[17] R. Carlin et al., "The Trigger of ZEUS, a Flexible System for a High Bunch Crossing Rate Collider", Nucl. Instr. Meth. A379 (1996) 542.

[18] W. H. Smith et al., "The ZEUS First Level Calorimeter Trigger", Nucl. Instr. Meth. A355 (1995) 278.

[19] G. P. Heath et al., ”The ZEUS First Level Tracking Trigger”, Nucl. Instr. Meth. A315 (991) 431.

[20] F. Sefkow et al., "Experience with the First Level Trigger of H1", IEEE Trans. Nucl. Sci. 42 (1995) 900.

[21] ATLAS Level-1 Trigger: Technical Design Report CERN/LHCC 98-14 (1998).

[22] J. Pliszka, G. Wrochna, "Estimate of Combined Muon/Calorimeter Rates", CERN CMS Note 1998/075 (1998). 
[23] B. G. Taylor, TTC Distribution for LHC Detectors, IEEE Trans. Nucl. Sci. 45, No. 3 (1998) 821.

[24] W. H. Smith, A. Taurok, J. Varela, C.-E. Wulz, "The CMS Trigger Control System", CERN CMS Note in preparation.

[25] W.H. Smith et al., "CMS Calorimeter Trigger Receiver System", Proc. of the Fourth Workshop on Electronics for LHC Experiments, Rome, Italy, Sept. 1998, CERN/LHCC/98-36 (1998) 321.

[26] S. Dasu et al., "CMS Calorimeter Regional Trigger Prototypes", Proc. of the Fifth Workshop on Electronics for LHC Experiments, Snowmass, Co, USA, Sept. 1999, CERN/LHCC/99-33 (1999) 309.

[27] G. P. Heath, "Trigger Processing Using Reconfigurable Logic in the CMS Calorimeter Trigger", Proc. of the 8th Pisa Meeting on Advanced Detectors, La Biodola, Isola d'Elba, Italy (2000), to be published in Nucl.Instr. Meth. A.

[28] M. Andlinger et al., "Pattern Comparator Trigger (PACT) for the Muon System of the CMS experiment", Nucl. Instr. Meth. A370 (1996) 389.

[29] F. Gasparini et al., "Bunch Crossing Identification at LHC Using a Meantimer Technique”, Nucl. Instr. Meth. A336 (1993) 91.

[30] R. Martinelli et al., "Design of the Track Correlator for the DTBX Trigger", CERN CMS Note 1999/007 (1999).

[31] I. D’Antone et al., "Track-Segment Sorting in the Trigger Server of a Barrel Muon-Station in CMS", CERN CMS Technical Note 1996/078 (1996).

[32] A. Kluge, T. Wildschek, "The Hardware Muon Trigger Track Finder Processor in CMS - Specification and Method", CERN CMS Note 1997/091 (1997).

[33] A. Kluge, T. Wildschek, "The Hardware Muon Trigger Track Finder Processor in CMS - Architecture and Algorithm”, CERN CMS Note 1997/092 (1997).

[34] A. Kluge, T. Wildschek, 'The Hardware Muon Trigger Track Finder Processor in CMS - Prototype and Final Implementation", CERN CMS Note 1997/093 (1997).

[35] J. Erö, ”New Approach for the CMS Muon Trigger Track Finder Processor", Proc. of the Fifth Workshop on Electronics for LHC Experiments, Snowmass, Co, USA, Sept. 1999, CERN/LHCC/99-33 (1999) 309.

[36] M. Kloimwieder, "Improving the $\eta$-Assignment of the DTBX-Based First Level Regional Muon Trigger", CERN CMS Note 1999/054 (1999).

[37] J. Hauser, "Primitives for the CMS Cathode Strip Muon Trigger", Proc. of the Fifth Workshop on Electronics for LHC Experiments, Snowmass, Co, USA, Sept. 1999, CERN/LHCC/99-33 (1999) 304.

[38] T.Y. Ling, "Front-End Electronics of the CMS Endcap Muon System", Proc. of the Fourth Workshop on Electronics for LHC Experiments, Rome, Italy, Sept. 1998, CERN/LHCC/98-36 (1998) 262.

[39] D. Acosta et al., "The Track-Finder Processor for the Level-1 Trigger of the CMS Endcap Muon System", Proc. of the Fifth Workshop on Electronics for LHC Experiments, Snowmass, Co, USA, Sept. 1999, CERN/LHCC/99-33 (1999) 318.

[40] N. Neumeister, P. Porth, H. Rohringer, "Simulation of the Global Muon Trigger", CERN CMS Internal Note 1997/023.

[41] A. Taurok, 'Implementation and Synchronisation of the First Level Global Trigger for the CMS Experiment at LHC”, CERN CMS Note 2000/057 (2000). 